\title{
The ThRombolysis in UnconTrolled Hypertension (TRUTH) protocol: an observational study on treatment strategy of elevated blood pressure in stroke patients eligible for IVT
}

\author{
T. P. Zonneveld ${ }^{1}$, A. Algra ${ }^{2,3}$, D. W. J. Dippel ${ }^{5}$, L. J. Kappelle ${ }^{4}$, R. J. van Oostenbrugge ${ }^{6}$, Y. B. W. E. M. Roos ${ }^{1}$, \\ M. J. Wermer ${ }^{7}$, H. B. van der Worp ${ }^{4}$, P. J. Nederkoorn' ${ }^{1}$, N. D. Kruyt ${ }^{*}$ and for the TRUTH investigators
}

\begin{abstract}
Background: Intravenous thrombolysis (IVT) with (recombinant) tissue plasminogen activator is an effective treatment in acute ischemic stroke. However, IVT is contraindicated when blood pressure is above 185/110 mmHg, because of an increased risk on symptomatic intracranial hemorrhage. In current Dutch clinical practice, two distinct strategies are used in this situation. The active strategy comprises lowering blood pressure with antihypertensive agents below these thresholds to allow start of IVT. In the conservative strategy, IVT is administered only when blood pressure drops spontaneously below protocolled thresholds. A retrospective analysis in two recent stroke trials showed a non-significant signal towards better functional outcome in the active group; robust evidence for either strategy, however, is lacking. We hypothesize that (I) the active strategy leads to a better functional outcome three months after acute ischemic stroke. Secondary hypotheses are that this effect occurs despite (II) increasing the number of symptomatic intracranial hemorrhages, and could be attributable to (III) a higher rate of IVT treatments and (IV) a shorter door-to-needle time.
\end{abstract}

Methods and design: The TRUTH is a prospective, observational, cluster-based, parallel group follow-up study; in which participating centers continue their current local treatment guidelines. Outcomes of patients admitted to centers with an active will be compared to those admitted to centers with a conservative strategy. The primary outcome is functional outcome on the modified Rankin Scale at three months. Secondary outcomes are symptomatic intracranial hemorrhage, IVT treatment and door-to-needle time. We based our sample size estimate on an ordinal analysis of the mRS with the "proportional odds" model. With the aforementioned signal observed in a recent retrospective study in these patients as an estimate of the effect size and with alpha $0 \cdot 05$, this analysis would have an $80 \%$ power with a total number of 600 patients. Corrections for expected imbalance in group size and clustering effects resulted in a sample size of 1235 patients.

Discussion: The TRUTH is the first large prospective study specifically studying IVT-candidates with elevated blood pressure, and has the potential to change clinical practice and optimize acute stroke care in these patients.

Keywords: Ischemic stroke, Intravenous thrombolysis, Blood pressure treatment

\footnotetext{
* Correspondence: ndkruyt@gmail.com

${ }^{7}$ Department of Neurology, Leiden University Medical Center, Leiden, The Netherlands

Full list of author information is available at the end of the article
}

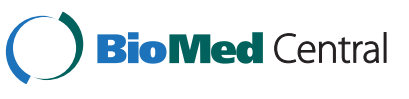

(c) 2015 Zonneveld et al. Open Access This article is distributed under the terms of the Creative Commons Attribution 4.0 International License (http://creativecommons.org/licenses/by/4.0/), which permits unrestricted use, distribution, and reproduction in any medium, provided you give appropriate credit to the original author(s) and the source, provide a link to the Creative Commons license, and indicate if changes were made. The Creative Commons Public Domain Dedication waiver (http://creativecommons.org/publicdomain/zero/1.0/) applies to the data made available in this article, unless otherwise stated. 


\section{Background}

\section{Elevated pre-treatment blood pressure}

Intravenous thrombolysis (IVT) with recombinant tissue plasminogen activator (rtPA) is an effective treatment for acute ischemic stroke [1]. Unfortunately, the number of stroke patients treated with rtPA is still limited [2]. One of the main reasons that patients are withheld IVT is a pre-treatment blood pressure (BP) above systolic BP of $185 \mathrm{mmHg}$ or a diastolic BP $>110 \mathrm{mmHg}$. These BP thresholds were introduced in the National Institute of Neurological Disorders and Stroke (NINDS) rtPA Stroke Study [3], and were based on pilot studies in which high diastolic BP levels were associated with an increased risk of symptomatic intracerebral hemorrhage $(\mathrm{sICH})$ after treatment with rtPA [4]. In a recent post-hoc analysis in the PRomoting ACute Thrombolysis in Ischemic StrokE (PRACTISE) [5] trial and the Preventive Antibiotics in Stroke Study (PASS) [6], the prevalence of elevated pretreatment BP in patients otherwise eligible for IVT was substantial: $20 \%$ [7], which is similar to that in a previous retrospective study [8].

\section{Treatment strategies (active vs. conservative)}

In current Dutch clinical practice, and according to national guidelines, neurologists can use two distinct strategies in managing elevated pre-treatment BP in patients otherwise eligible for IVT [9].

The first strategy is active BP lowering with antihypertensive agents, aiming to lower BP below the thresholds to allow IVT. This strategy is in line with recommendations in current international guidelines that BP should be carefully lowered in these patients (Class of evidence: Class IV [10], and Level of Evidence B [11]). However, these recommendations are based on expert opinion only, and several critical remarks can be made regarding this strategy. First, patients with elevated pre-treatment BP were included in the larger IV rtPA trials only if they reached BP thresholds with oral or sublingual antihypertensive medication (e.g. NINDS [3], ATLANTIS [12] and ECASS-III [13]). Therefore, little is known about the efficacy and safety of IVT in patients who require intravenous antihypertensive medication. Secondly, despite lowering BP below IVT thresholds, the risk on sICH could still be increased. Indeed, one retrospective study found an increased relative risk of 2 . 47 (95\% confidence interval, $1 \cdot 15$ to $5 \cdot 28$ ) on $\mathrm{sICH}$ in patients treated for elevated pre-treatment BP [14]. Two similar studies found similar results but the associations were not statistically significant $[15,16]$. Because all three studies were retrospective and relatively small $(n=510$, 427 and 178 respectively), it is difficult to draw any sound conclusions. Finally, iatrogenic BP lowering in a state of failing cerebral autoregulation could compromise perfusion of the already ischemic penumbra, with subsequent neurological deterioration [17].
The second strategy concerning pre-treatment increased blood pressure is to measure and wait if the blood pressure drops spontaneously. This avoids the possible disadvantages of active BP lowering as mentioned above and is current clinical practice in several Dutch centers, despite international guidelines that advocate an active strategy. In these centers antihypertensive agents are not routinely administered, and IVT is administered only when the BP drops spontaneously below the recommended thresholds. The major disadvantage of this strategy is that fewer patients end up being treated with IVT, when there is no spontaneous decrease in BP [7]. Another disadvantage could be an increase of the door-to-needle time (DNT) while waiting for a spontaneous drop in BP, while an active strategy is associated with only a modest increase in the DNT [18]. However, in a recent study this increase in DNT was not found [7].

\section{Clinical practice}

In contrast to other European countries, Dutch stroke guidelines do not recommend active lowering of blood pressure to date. In order to clarify current clinical practice, and in order to legitimize the proposed trial, we performed an online questionnaire among vascular neurologists in Dutch centers administering IVT. Questionnaires were sent to neurologists in 81 centers and 75 responded (93\%). Of these, 51 (68\%) reported to use an active - and 17 (23\%) a conservative strategy. The remaining 7 centers reported not to have a uniform strategy (unpublished data).

\section{Aim}

The aim of our study is to elucidate whether we should actively lower elevated pre-treatment BP in patients eligible for IVT.

\section{Methods}

Hypothesis

We hypothesize that in patients with a pre-treatment BP above $185 / 110 \mathrm{mmHg}$ and otherwise eligible for IVT an active BP lowering strategy leads to [1] a better functional outcome at three months than a conservative watch and wait strategy. Secondary hypotheses are that this effect occurs despite [2] increasing the proportion of symptomatic intracranial hemorrhages, and could be attributable to [3] a higher rate of IVT treatments and [4] a shorter door-to-needle time.

\section{Design}

The ThRombolysis in UnconTrolled Hypertension (TRUTH) is an observational, prospective, multicenter, cluster-based, parallel group follow-up study. 


\section{Patient population}

All adult ischemic stroke patients with elevated pretreatment $\mathrm{BP}$ as the only contraindication to delay or withhold treatment with IVT.

\section{Treatment strategy}

Participating centers will continue their current local treatment guideline (either active or conservative strategy). Both the active and conservative strategy have to be formalized in local guidelines, and have to be endorsed by all neurologists executing acute stroke care. These local guidelines do not have to match specific preset criteria, for example regarding route of administration or dosage schemes of antihypertensive agents. Centers are allowed to change their strategy during the course of the study. Patients will receive either strategy, depending on to which center they are admitted.

\section{Intra-arterial therapy}

Treatment with intra-arterial therapy (IAT) is not an exclusion criterion, and in all our analyses we will adjust for this factor. Patients receiving IAT without prior IVT because of elevated BP are also eligible for the TRUTH study as the same contra-indication applies for these patients.

Eleven of the 36 participating centers are currently performing IAT, and ten of these use an active treatment strategy. However, patients enrolled in non-interventional centers eligible for IAT are likely to be referred to an intervention center. Therefore, we expect no major difference in the number of patients receiving IAT between both groups.

\section{Outcome measures}

The primary outcome is the functional outcome on the modified Rankin Scale (mRS) at 90 days assessed with a telephone interview by trained research nurses blinded for the treatment strategy. Secondary outcomes are symptomatic intracranial hemorrhage (sICH), defined as any CT-documented hemorrhage accompanied by clinical deterioration identified as $\geq 4$ points increase on the NIHSS compared with the best recorded NIHSS since admission (ECASS II criteria) [19], the rate of patients receiving IVT, and the door-to-needle time recorded as recently defined by Kruyt et al. [20].

\section{Statistical analysis}

The primary analysis will be an ordinal analysis of the seven categories on the mRS, by means of the proportional odds model [21]. In this model, it is assumed that the odds ratio is proportional across all possible dichotomization levels of the ordinal outcome, which will be formally tested with Rao's score test. To produce an effect estimate (odds ratio with corresponding $95 \%$ confidence interval (CI)) and to allow for covariate adjustment, ordinal logistic regression will be used. Secondly, to assess internal consistence, two fixed dichotomous analyses of the outcome on the mRS will also be performed (good outcome $0-1$ vs. poor outcome $2-6$, and favorable outcome $0-2$ vs. unfavorable 3-6). Secondary outcomes will be analyzed in a Poisson regression analysis (binary outcome; sICH, IVT) or linear regression analysis (continuous outcome; DNT). IVT and sICH rates will be reported as percentages with $95 \%$ CIs, DNTs will be reported as mean with standard deviation or median with interquartile range depending on its distribution.

\section{Sample size estimates}

We based our sample size on the ordinal analysis of the $\mathrm{mRS}$. With the increase in favorable outcome in the active group in the post-hoc analysis as an estimate of the effect size and with alpha $0 \cdot 05$, this analysis will have a power of $80 \%$ if we include 600 patients. The distribution between both groups will not be equal; we estimated a ratio of 3:1 in favor of the active strategy based on our aforementioned questionnaire. Since such a distribution leads to a 1.33 fold increase of the sample size in a regular chi-square test, we also applied this factor to our sample size estimate, resulting in an estimate of 798 patients.

Because of presumed similarity among patients within preexisting clusters, the variability in response and the power to detect true differences between arms are reduced in a clustered sample. We used the formula by Kerry and Bland to correct for this clustering effect [22]:

$$
\mathrm{DE}=1+\rho\left(\left\{\mathrm{cv}^{2}+1\right\} \mathrm{m}-1\right)
$$

The design effect (DE) is the factor that is ultimately applied to a standard sample size estimate to correct for the clustered design. First, we need to estimate the intracluster coefficient $(\rho)$, which is reflected by the ratio of the variance of a certain variable between and within clusters. With this intracluster coefficient, the mean number of patients per cluster $(\mathrm{m})$, and the coefficient of variation in cluster size (cv, to correct for imbalances in cluster size) [23], the DE is estimated. We used an intracluster coefficient of 0.015 in our calculation. This number was derived from the multicenter cluster-randomized PRACTISE trial [5], in which an ICC of 0.0154 was calculated using their outcome on thrombolysis percentages from 12 Dutch hospitals. We estimated the $\mathrm{cv}$ to be 0.50 , based on a similar observed coefficient in a study with cluster characteristics comparable to those we expect in our study [24]. Furthermore, we estimate the mean number of patients per cluster to be 30 . With these estimations, our design effect is 1.5475 and therefore our effective sample size will be 798 patients with an actual sample size of 1235 patients. 


\section{Feasibility}

In the Netherlands, 3760 patients in 86 hospitals were treated with IVT in 2012 [25]. Therefore, we estimate that elevated pre-treatment BP occurs in at least 750 IVT patients per year. In our aforementioned post-hoc analysis, we identified 231 patients with elevated pretreatment BP of whom 149 received IVT. Therefore, our best estimate of the number of patients in the Netherlands with elevated pre-treatment BP is 1163 per year $(231 \times 750 / 149)$.

We estimate the mean number of patients subjected to IVT per participating center per year to be 50 . Therefore, in the average center, elevated pre-treatment BP will occur at least 10 times each year. If we include patients for 4 consecutive years, we need 31 centers to participate. As treatment allocation is determined by center, refusal to participate on patients level will be negligible. A list of participating centers and local investigators is supplied in Appendix 1: Executive team.

\section{Discussion}

Elevated pre-treatment blood pressure (BP) is the most common potentially modifiable contraindication for intravenous thrombolysis (IVT). Current international guidelines recommend administering antihypertensive agents in order to lower BP below IVT thresholds. However, this recommendation is based on relatively small, retrospective studies that only report symptomatic intracranial hemorrhage $(\mathrm{sICH})$ as outcome. In contrast to other European countries, Dutch stroke guidelines do not recommend active lowering of blood pressure to date. In most Dutch centers antihypertensive agents are administered (active strategy), whereas in other centers they are not (conservative strategy). In an international Delphi study on contraindications for IVT, no consensus was reached concerning BP management [26]. In our opinion, it remains therefore unclear whether patients with elevated pre-treatment BP should be actively treated with antihypertensive agents or not. The TRUTH study is the first large prospective study specifically designed to elucidate this clinical question.

Following up on the post-hoc analysis in PRACTISE [5] and PASS [6], which revealed a non-significant trend towards better functional outcome in patients subjected to an active treatment strategy, several design options for further study on this clinical question were considered. A randomized design, with randomization either at patient level or at center level, was considered first. However, we deemed this to be unfeasible. Randomization at patient level would be highly susceptible to selection bias, as most treating physicians have a strong preference towards a certain strategy and would therefore be prone to only include specific patients. Besides, in this hyper acute setting where time is of essence, acquiring patient consent for randomization would cost too much time. Randomization at cluster level was deemed unfeasible, as most centers have formulated their treatment strategies in local guidelines and would be unwilling to participate in a trial that would allocate their center randomly to a either strategy.

An observational design is feasible because of the different treatment strategies used in Dutch clinical practice. In some centers, where no uniform strategy was yet defined, one of both strategies was adopted in order to participate in the study. Because of the small number of Dutch centers administering IVT in which no uniform strategy was defined (7 out of 75 in our questionnaire) and the possibility to participate in this study after adopting a uniform strategy, we judge the possibility of selection bias on center level to be very low. The observational design also allowed us to start relatively fast with subject enrollment, an important pragmatic argument in a field moving steadily towards the active strategy despite lack of evidence. Furthermore, we expect that our design results in a much higher participation rate at center level and a higher enrollment rate at patient level in comparison with a randomized design.

As a safety endpoint we used the ECASS-II definition of sICH (any CT-documented hemorrhage accompanied by clinical deterioration identified as $\geq 4$ points increase on the NIHSS compared with the best recorded NIHSS since admission). We used this definition because a recent study judged it to be more clinically relevant than most other definitions, in terms of its ability to identify hemorrhages that alter functional outcome three months after acute ischemic stroke [27].

We considered an ordinal analysis of the modified Rankin Scale (mRS) preferable to a binary (dichotomized) analysis for two reasons: First, an ordinal analysis is more efficient in models with a treatment effect present over the entire range of the outcome scale (as we expect in our study); because it retains all information captured by the outcome scale, an ordinal analysis improves study power in such models [28]. Secondly, multiple underlying mechanisms of an active strategy treatment effect might be present simultaneously (higher IVT rate, shorter door-to-needle time, higher $\mathrm{sICH}$ rate, iatrogenic penumbral hypoperfusion), and these could express themselves in different directions in different parts of the mRS spectrum. An ordinal analysis is therefore especially appropriate as it captures shifts in both directions (efficacy and safety) in a single analysis [29].

Overall, the TRUTH study is designed to improve clinical practice and optimize acute stroke care in IVT candidates with elevated blood pressure.

\section{Trial status}

Recruitment started in April 2015 and is ongoing (85 participants recruited as of November 1st, 2015). 


\section{Ethics}

Written informed consent will be obtained from each patient or legal representative. The patient information describes the purpose and design of the study, and the procedures for recording clinical information and 3-month follow-up. Collection of consent and study enrollment do not have to be done before either treatment strategy is carried out, since the strategy in question is standard of practice in that center. The study protocol, patient information and enrollment procedure were assessed and approved by the Medical Ethics Committee and Review Board of the Academic Medical Center in Amsterdam. Local scientific and ethical committees of each participating center will perform further assessments of local feasibility.

\section{Appendix 1: TRUTH Investigators}

Principal investigators: N.D. Kruyt, MD PhD; P.J. Nederkoorn, MD, PhD.

Steering committee: A. Algra, MD, PhD; D.W.J. Dippel, $\mathrm{MD}, \mathrm{PhD}$; L.J. Kappelle, $\mathrm{MD}, \mathrm{PhD}$; R.J. van Oostenbrugge, MD, PhD; Y.B.W.E.M. Roos, MD, PhD; M.J. Wermer, MD, PhD; H.B. van der Worp, MD, PhD.

Participating centers (local investigator) as of November 2015: Active: Leiden University Medical Center, Leiden (N.D. Kruyt); Erasmus University Medical Center, Rotterdam (D.W.J. Dippel); University Medical Center Utrecht, Utrecht (H.B. van der Worp); Maastricht University Medical Center Plus, Maastricht (R.J. van Oostenbrugge); Admiral de Ruyter Hospital, Goes (E.W. Peters); Atrium Medical Center, Heerlen (A. Schreuder); Bethesda Hospital, Hoogeveen (J.H. Kwant); Elkerliek Hospital, Helmond (G.S. Grooters); Hospital Gelderse Vallei, Ede (J.M.P. Rovers); Groene Hart Hospital, Gouda (K. de Gans); Maasstad Hospital, Rotterdam (R. Saxena); Maxima Medisch Centrum, Eindhoven (B.C.A.M. van Gin neken); Medical Center Haaglanden Westeinde, The Hague (K. Jellema); Medical Center Leeuwarden, Leeuwarden (W.J. Schuiling); Medisch Spectrum Twente, Enschede (P.J.A.M. Brouwers); Radboud UMC, Nijmegen (E. Richard); Reinier de Graaf Gasthuis, Delft (L.A.M. Aerden); Rijnland Hospital, Leiderdorp (E.L.L.M. de Schryver); Rijnstate, Arnhem (S.E. Vermeer); St. Elisabeth Hospital, Tilburg (J.H. van Tuijl); St. Franciscus Hospital, Rotterdam (F.H. Vermeij); St. Jans Gasthuis, Weert (H. Lövenich); Hospital St. Jansdal, Harderwijk (A. Bijl-van Dijk); Slotervaart Hospital, Amsterdam (F.H.M. Spaander); VieCuri, Venlo (A.M.H.G. van der Heijden); Vlietland Hospital, Schiedam (C.L. Alblas). Conservative: Academic Medical Center Amsterdam, Amsterdam (P.J. Nederkoorn); Albert Schweitzer Hospital, Dordrecht (H. Kerkhoff); Kennemer Gasthuis, Haarlem (F. de Beer); Rode Kruis Hospital, Beverwijk (W.D.M. van der Meulen); VU University Medical Center, Amsterdam (M.C. Visser); Medical Center Alkmaar,
Alkmaar (P. Halkes); Onze Lieve Vrouwe Gasthuis, Amsterdam (V.I.H. Kwa); St. Lucas Andreas Hospital, Amsterdam (R.M. van den Berg - Vos); Tergooiziekenhuizen, Hilversum (J.R. de Kruijk); Westfriesgasthuis, Hoorn (T.C. van der Ree).

\begin{abstract}
Abbreviations
BP: blood pressure; DE: design effect; DNT: door-to-needle time; IAT: intra-arterial therapy; IVT: intravenous thrombolysis; mRS: modified rankin scale; NINDS: National Institute of Neurological Disorders and Stroke; PASS: preventive antibiotics in stroke study; PRACTISE: PRomoting ACute Thrombolysis in Ischemic StrokE; rtPA: recombinant tissue plasminogen activator; sICH: symptomatic intracranial hemorrhage; TRUTH: thrombolysis and uncontrolled hypertension.
\end{abstract}

\section{Competing interests}

The authors declare that they have no competing interests.

Authors' contributions

NDK and PJN are the co-Principal Investigators of the study and contributed equally. TPZ wrote the first draft. All authors contributed to the study design and final manuscript.

\section{Acknowledgements}

We would like to acknowledge Dr Jonathan M. Coutinho for substantial contributions to the conception and design of our study. Dr Coutinho was supported by The Netherlands Organisation for Scientific Research, grant number 021.001.045 and the Dutch Thrombosis Society, grant number 2012-2.

\section{Funding}

The TRUTH study is funded by a grant of NutsOhra Foundation (project 1302-062)

Local TRUTH investigators are listed in Appendix 1

\section{Author details}

${ }^{1}$ Department of Neurology, Academic Medical Center, Amsterdam, The Netherlands. ${ }^{2}$ Department of Clinical Epidemiology, Leiden University Medical Center, Leiden, The Netherlands. ${ }^{3}$ The Julius Center, University Medical Center Utrecht, Utrecht, The Netherlands. ${ }^{4}$ Department of Neurology and Neurosurgery, Brain Center Rudolf Magnus, University Medical Center Utrecht, Utrecht, The Netherlands. ${ }^{5}$ Department of Neurology, Erasmus Medical Center, Rotterdam, The Netherlands. 'Department of Neurology, Maastricht UMC+, Maastricht, The Netherlands. 'Department of Neurology, Leiden University Medical Center, Leiden, The Netherlands.

Received: 31 August 2015 Accepted: 11 November 2015 Published online: 23 November 2015

\section{References}

1. Wardlaw JM, Murray V, Berge E, del Zoppo G, Sandercock P, Lindley RL, et al. Recombinant tissue plasminogen activator for acute ischaemic stroke: an updated systematic review and meta-analysis. Lancet. 2012:379(9834):2364-72.

2. Moradiya Y, Crystal H, Valsamis H, Levine SR. Thrombolytic utilization for ischemic stroke in US hospitals with neurology residency program. Neurology. 2013;81(23):1986-95.

3. Tissue plasminogen activator for acute ischemic stroke. The National Institute of Neurological Disorders and Stroke rt-PA Stroke Study Group. N Engl J Med. 1995:333(24):1581-7.

4. Levy DE, Brott TG, Haley Jr EC, Marler JR, Sheppard GL, Barsan W, et al. Factors related to intracranial hematoma formation in patients receiving tissue-type plasminogen activator for acute ischemic stroke. Stroke. 1994 25(2):291-7

5. Dirks M, Niessen LW, van Wijngaarden JDH, Koudstaal PJ, Franke $C L$, van Oostenbrugge RJ, et al. Promoting thrombolysis in acute ischemic stroke. Stroke. 2011:42(5):1325-30.

6. Westendorp WF, Vermeij J-D, Zock E, Hooijenga IJ, Kruyt ND, Bosboom HJLW, et al. The Preventive Antibiotics in Stroke Study (PASS): a pragmatic randomised open-label masked endpoint clinical trial. Lancet. 2015. 
7. Dirks M, Zonneveld TP, Dippel DWJ, Nederkoorn PJ, van de Beek D, van Oostenbrugge RJ, et al. Elevated pretreatment blood pressure and IV thrombolysis in stroke. Neurology. 2015;84(14):1419-25.

8. Kleindorfer D, Kissela B, Schneider A, Woo D, Khoury J, Miller R, et al. Eligibility for recombinant tissue plasminogen activator in acute ischemic stroke: a population-based study. Stroke. 2004;35(2):e27-9.

9. Bauer A, Limburg M, Visser MC. Variation in clinical practice of intravenous thrombolysis in stroke in the Netherlands. Cerebrovasc Dis Extra. 2013;3(1):74-7.

10. Ringleb PA, Bousser MG, Ford G, Bath P, Brainin M, Caso V, et al. Guidelines for management of ischaemic stroke and transient ischaemic attack 2008. Cerebrovasc Dis. 2008;25(5):457-507.

11. Jauch EC, Saver JL, Adams Jr HP, Bruno A, Connors JJB, Demaerschalk BM, et al. Guidelines for the early management of patients with acute ischemic stroke: a guideline for healthcare professionals from the American Heart Association/American Stroke Association. Stroke. 2013;44(3):870-947.

12. Clark WM, Wissman S, Albers GW, Jhamandas JH, Madden KP, Hamilton S. Recombinant tissue-type plasminogen activator (Alteplase) for ischemic stroke 3 to 5 hours after symptom onset. The ATLANTIS Study: a randomized controlled trial. Alteplase Thrombolysis for Acute Noninterventional Therapy in Ischemic Stroke. JAMA. 1999;282(21):2019-26.

13. Hacke W, Kaste M, Bluhmki E, Brozman M, Dávalos A, Guidetti D, et al. Thrombolysis with alteplase 3 to 4.5 hours after acute ischemic stroke. N Engl J Med. 2008:359(13):1317-29.

14. Tsivgoulis G, Frey JL, Flaster M, Sharma VK, Lao AY, Hoover SL, et al. Pre-tissue plasminogen activator blood pressure levels and risk of symptomatic intracerebral hemorrhage. Stroke. 2009;40(11):3631-4.

15. Martin-Schild S, Hallevi H, Albright KC, Khaja AM, Barreto AD, Gonzales NR, et al. Aggressive blood pressure-lowering treatment before intravenous tissue plasminogen activator therapy in acute ischemic stroke. Arch Neurol. 2008; 65(9):1174-8.

16. Kellert L, Rocco A, Sykora M, Hacke W, Ringleb PA. Frequency of increased blood pressure levels during systemic thrombolysis and risk of intracerebral hemorrhage. Stroke. 2011;42(6):1702-6.

17. Owens WB. Blood pressure control in acute cerebrovascular disease. J Clin Hypertens (Greenwich). 2011;13(3):205-11.

18. Skolarus LE, Scott PA, Burke JF, Adelman EE, Frederiksen SM, Kade AM, et al. Antihypertensive Treatment Prolongs Tissue Plasminogen Activator Door-toTreatment Time: Secondary Analysis of the INSTINCT Trial. Stroke. 2012.

19. Hacke W, Kaste M, Fieschi C, von Kummer R, Davalos A, Meier D, et al. Randomised double-blind placebo-controlled trial of thrombolytic therapy with intravenous alteplase in acute ischaemic stroke (ECASS II). Second European-Australasian Acute Stroke Study Investigators. Lancet. 1998; 352(9136):1245-51.

20. Kruyt ND, Nederkoorn PJ, Dennis M, Leys D, Ringleb PA, Rudd AG, et al. Door-to-needle time and the proportion of patients receiving intravenous thrombolysis in acute ischemic stroke: uniform interpretation and reporting. Stroke. 2013;44(11):3249-53.

21. Whitehead J. Sample size calculations for ordered categorical data. Stat Med. 1993:12(24):2257-71.

22. Killip S, Mahfoud Z, Pearce K. What is an intracluster correlation coefficient? crucial concepts for primary care researchers. Ann Fam Med. 2004;2(3):204-8,

23. Eldridge SM, Ashby D, Kerry S. Sample size for cluster randomized trials: effect of coefficient of variation of cluster size and analysis method. Int J Epidemiol. 2006;35(5):1292-300.

24. Burns T, Kendrick T. Care of long-term mentally ill patients by British general practitioners. Psychiatr Serv. 1997;48(12):1586-8.

25. Inspectie voor de Gezondheidszorg MVV, Welzijn en Sport. Het resultaat telt Ziekenhuizen 2012. 2014.

26. Dirks M, Niessen LW, Koudstaal PJ, Franke CL, Van Oostenbrugge RJ, Dippel DWJ. Intravenous thrombolysis in acute ischaemic stroke: from trial exclusion criteria to clinical contraindications. An international Delphi study. J Neurol Neurosurg Psychiatry. 2007;78(7):685-9.

27. Rao NM, Levine SR, Gornbein JA, Saver JL. Defining clinically relevant cerebral hemorrhage after thrombolytic therapy for stroke: analysis of the National Institute of Neurological Disorders and Stroke tissue-type plasminogen activator trials. Stroke. 2014;45(9):2728-33.

28. Saver $\mathrm{JL}$, Gornbein J. Treatment effects for which shift or binary analyses are advantageous in acute stroke trials. Neurology. 2009;72(15):1310-5.

29. Bath PMW, Lees KR, Schellinger PD, Altman H, Bland M, Hogg C, et al. Statistical analysis of the primary outcome in acute stroke trials. Stroke. 2012;43(4):1171-8.

\section{Submit your next manuscript to BioMed Central and take full advantage of:}

- Convenient online submission

- Thorough peer review

- No space constraints or color figure charges

- Immediate publication on acceptance

- Inclusion in PubMed, CAS, Scopus and Google Scholar

- Research which is freely available for redistribution

Submit your manuscript at www.biomedcentral.com/submit 